\title{
Searching for the odderon in ultraperipheral proton-ion collisions at the $\mathrm{LHC}$
}

\author{
L. A. Harland-Lang, ${ }^{1}$ V. A. Khoze, ${ }^{2,3}$ A. D. Martin, ${ }^{2}$ and M. G. Ryskin ${ }^{2,3}$ \\ ${ }^{1}$ Rudolf Peierls Centre, Beecroft Building, Parks Road, Oxford, OX1 3PU \\ ${ }^{2}$ Institute for Particle Physics Phenomenology, University of Durham, Durham, DH1 3 LE \\ ${ }^{3}$ Petersburg Nuclear Physics Institute, NRC Kurchatov Institute, Gatchina, St. Petersburg, 188300, Russia
}

(Received 10 December 2018; published 20 February 2019)

\begin{abstract}
We explore the possibility of observing odderon exchange in proton-ion collisions at the LHC, via the ultraperipheral photoproduction of C-even mesons. As well as the signal, we consider in detail the principle backgrounds, due to QCD-initiated production (i.e., double pomeron exchange) and $\gamma \gamma$ fusion. We find that while the photon-initiated background is dominant at very small momentum transfer, this can be effectively removed by placing a reasonable cut on the transverse momentum of the produced meson. In the case of QCD-initiated production, we show this is in general strongly suppressed by the small probability of no additional particle production in the central detector, namely the survival factor. In some scenarios, this suppression is sufficient to permit the observation of odderon exchange in $\mathrm{Pb}-p$ collisions in a clean environment, or else to place bounds on this. We in addition identify the cases of $\pi^{0}$ and $\eta(548)$ production as particularly promising channels. Here, the QCD-initiated background is absent for $\pi^{0}$ due to isospin conservation and very small for $\eta(548)$ due to its dominantly flavor octet nature and odd parity.
\end{abstract}

DOI: 10.1103/PhysRevD.99.034011

\section{INTRODUCTION}

The TOTEM experiment at the LHC [1] has recently published the results of the first high precision measurement at $\sqrt{s}=13 \mathrm{TeV}$ of the ratio of the real-to-imaginary parts of the forward elastic $p p$-amplitude, $\rho=\operatorname{Re} A / \operatorname{Im} A$. The measured value of $\rho=0.09-0.10$ is smaller than that predicted by the conventional COMPETE parametrization [2], which gives $\rho=0.135$. This may indicate either a slower increase of the total cross section at higher energies or a manifestation of the odd-signature amplitude, which is not included in the COMPETE parametrization.

This TOTEM result has generated renewed interest [3-19] in the long-standing issue of establishing the existence of the odd-signature partner of the pomeron, the so-called odderon. This was first introduced in the early seventies in the framework of asymptotic theories [20,21], and since then has been the subject of intensive theoretical discussion, see e.g., Refs. [22-25] for reviews. This oddsignature exchange, which depends only weakly on energy, is a firm prediction of QCD [26,27] (see e.g., Ref. [28] for a more recent textbook discussion). In Ref. [29] it was shown that in the perturbative regime there exists a colorless C-odd

Published by the American Physical Society under the terms of the Creative Commons Attribution 4.0 International license. Further distribution of this work must maintain attribution to the author(s) and the published article's title, journal citation, and DOI. Funded by SCOAP. $t$-channel state, formed by three gluons, with an intercept $\alpha_{\text {Odd }}=1$.

In this paper we consider the possibility of observing an odderon exchange at the LHC via the semi-exclusive production of a $\mathrm{C}$-even meson $M$ in ultraperipheral heavyion-proton collisions, that is via the subprocess

$$
\gamma+p \rightarrow M+X
$$

where $X$ denotes the dissociation product of the proton, and the photon is emitted elastically from the ion. This subprocess is shown in Fig. 1(a), while the corresponding production process in $p \mathrm{~Pb}$ collisions is shown in Fig. 1(b), see Ref. [6] for recent work. In this case the signal is significantly enhanced by the photon flux from the ion by $\sim Z^{2}$, and as we will see the expected cross sections are certainly within reach at the LHC. However, for a full analysis it is essential to provide a proper consideration of the potential background contributions to this process, due either to double pomeron exchange, or $\gamma \gamma$ fusion. In reality, it is the magnitude of these backgrounds, rather than necessarily the signal size, that determines the level at which we could expect to observe or put new limits on the odderon contribution. We therefore present in this paper the first complete calculation of both the expected odderon signal and background contributions for C-even meson production at the LHC. As we shall discuss further below, an evaluation of the double pomeron exchange background requires a careful treatment of the proton-ion or ion-ion 




(a)

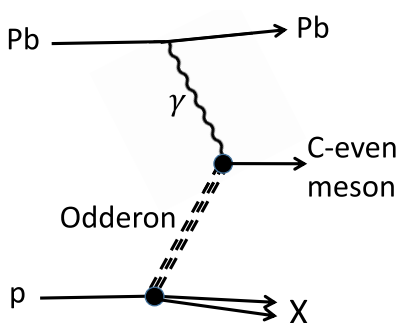

(b)
FIG. 1. The odderon exchange contribution to (a) photoproduction of C-even mesons and (b) exclusive ultraperipheral C-even meson production in $\mathrm{Pb}-p$ collisions.

collision process. We will see that, while in ion-ion collisions the background from $\gamma \gamma$ is overwhelming, in proton-ion collisions it is expected to be under better control.

The outline of this paper is as follows. In Sec. II we briefly summarize the current experimental situation with regards to odderon exchange, with emphasis on the phenomenology of C-even meson photoproduction. In Sec. III we present some general considerations for the conversion of the $\gamma p$ subprocess cross section to the case of $p \mathrm{~Pb}$ collisions, and translate the existing HERA results to expected upper limits on the odderon signal for $\pi^{0}, f_{2}$ and $\eta$ production at the LHC. In Sec. IV we present a phenomenological calculation of meson production via odderonexchange, concentrating on the $\pi^{0}$ case for concreteness. In Sec. $\mathrm{V}$ we discuss and provide a quantitative calculation of the background from $\gamma \gamma$ fusion. In Sec. VI we discuss and provide a quantitative calculation of the background from double pomeron exchange. In Sec. VII we discuss the reducible background arising from the radiative decay of vector mesons. In Sec. VIII we present a detailed numerical analysis of the signal and background cross sections for the cases of $f_{2}, \pi^{0}, \eta$ and $\eta_{c}$ production. Finally, in Sec. IX we conclude.

\section{SEARCHING FOR EVIDENCE OF ODDERON EXCHANGE}

Although in QCD we expect both pomeron and odderon exchange to contribute to scattering processes, the pre-LHC experimental quest for the odderon has proven to be quite a challenging task, see for instance Refs. [22,24,25,30] for reviews. In particular, the contribution from odderon exchange to elastic $p p$-scattering is predicted to be rather small, see e.g., Refs. [31,32], providing only a small correction to the dominant even-signature pomeron exchange. Moreover, due to screening effects this contribution is expected to decrease with increasing energy, see Refs. [4,31-33].

The possibility of probing the odderon via the high energy exclusive photoproduction of C-even mesons, which can be mediated by this odd-signature exchange, has a long history, see e.g., Refs. [34-41]. In particular, above the very low transverse momenta region where the major contribution comes from photon exchange, the odderon may dominate, see Fig. 1(a). The cross sections for light C-even meson $\left(\pi^{0}, \eta, f_{2}\right)$ photoproduction were evaluated e.g., in Refs. [35,37,39], by applying a perturbative QCD (PQCD) treatment with an effective cutoff applied to regulate the contribution from the infrared region.

The expected rates become rather large when break up of the target proton is permitted. In particular, an odderoninduced cross section of $\sim 300 \mathrm{nb}$ has been predicted for the $\gamma p \rightarrow \pi^{0}+X$ reaction [37], and about $21 \mathrm{nb}$ for the $\gamma p \rightarrow f_{2}+X$ case [39], at $\sqrt{s}=20 \mathrm{GeV}$. Since the $\eta$ meson belongs to the same multiplet as the pion we may expect more or less the same cross section for $\gamma p \rightarrow \eta+X$ photoproduction. More precisely, the rate will be a little lower due to the branching ratio of the considered $\eta$-decay and the smaller $u$-quark fraction in the wave function of the $\eta$ meson.

Searches for the odderon in the high energy photoproduction of $\mathrm{C}$-even mesons were performed at HERA at $\sqrt{s} \simeq 200 \mathrm{GeV}$ [42-44]. No signal was observed, and upper limits of $39 \mathrm{nb}$ for $\pi^{0}$ and $16 \mathrm{nb}$ for $f_{2}$ production, were set at the $95 \%$ confidence level, in conflict with these higher predictions. However, the results in Refs. [38,39] are only based on a leading order calculation in $\alpha_{S}$. Moreover, the major contribution in the corresponding integrals comes from the infrared domain (especially for the $\pi^{0}$ ), where it should be suppressed by confinement. In addition, the results are highly sensitive to the details of the proton wave function. For example, in the extreme limit of a proton formed by a quark and a pointlike diquark, the odderon to proton coupling, $g_{\mathrm{Op}}$, in fact vanishes (see e.g., Refs. [31,32,45]).

Away from this limit, the odderon-proton coupling is nonetheless expected to be small. This in fact highlights one of the general advantages of using inelastic photoproduction to search for odderon exchange, namely that the corresponding amplitude is proportional to the first power of the small odderon coupling $g_{\mathrm{Op}}$, while in elastic $p p$ scattering the amplitude is proportional to $g_{\mathrm{Op}}^{2}$. Moreover, by selecting events with larger meson transverse momenta, $k_{t}$, we can limit the sensitivity to the nonperturbative regime and corresponding infrared cutoff. As discussed in detail in Sec. IV, a PQCD based calculation suggests that a lower, but still sufficiently large cross section of

$$
\sigma(\gamma+p \rightarrow M+X) \sim 0.5-5 \mathrm{nb},
$$

is reasonable. As a result in $p \mathrm{~Pb}$ collisions we expect a cross section

$$
\sigma(p+\mathrm{Pb} \rightarrow X+M+\mathrm{Pb}) \sim 0.1-1 \mu \mathrm{b},
$$

for odderon-mediated photoproduction at the LHC via the semi-exclusive central process, which we will denote as 
CEP* in what follows. We emphasize that these estimates will serve as guidance when assessing the potential for observing odderon exchange at the LHC. However, in reality, the HERA upper limits on the photoproduction cross sections will provide the clearest indication of the possibility for $p \mathrm{~Pb}$ collisions at the LHC to observe the odderon. The emphasis below will be on calculating the size of the other contributing backgrounds, as is mandatory in a complete assessment of the LHC discovery potential.

\section{ODDERON EXCHANGE IN $p$ Pb COLLISIONS: GENERAL CONSIDERATIONS}

The odderon-exchange cross section for the production of a meson $M$ in $p \mathrm{~Pb}$ collisions can we written as

$$
\frac{\mathrm{d} \sigma^{p \mathrm{~Pb}}}{\mathrm{~d} Y_{M}} \sim \frac{\mathrm{d} N_{\gamma}}{\mathrm{d} Y_{M}} \sigma^{\gamma p},
$$

where $N_{\gamma}$ is the photon flux due to the lead ion and $Y_{M}$ is the meson rapidity. Here, we have used the fact that the cross section due to odderon exchange is expected to be independent of the $\gamma p$ energy, and neglected the rather smaller impact of the $p \mathrm{~Pb}$ survival factor. The advantage of using heavy ions is the significant enhancement of the photon flux; for lead the coherent $\gamma$-flux (i.e., with the lead ion remaining intact) is enhanced by $Z^{2}=82^{2}$. More precisely, the $\gamma$-flux reads (see e.g., Refs. [46-48])

$$
\frac{\mathrm{d} N_{\gamma}}{\mathrm{d} Y_{M}} \simeq \frac{\alpha_{\mathrm{QED}}}{\pi} \int \frac{q_{\perp}^{2}}{\left(q_{\perp}^{2}+\left(x m_{p}\right)^{2}\right)^{2}} F_{Z}\left(Q^{2}\right)^{2} \mathrm{~d} q_{\perp}^{2},
$$

where $m_{p}$ is the proton mass, $F_{Z}\left(Q^{2}\right)$ is the ion form factor, and $x$ is the momentum fraction carried by the photon. As an example, if we consider $\eta$ production in $p \mathrm{~Pb}$ collisions at $\sqrt{s}_{n n}=8.8 \mathrm{TeV}$ (corresponding to the maximum collision energy at the LHC), then at central rapidity $Y_{M}=0$ we have

$$
x \sim \frac{M_{\eta}}{\sqrt{s}}=6.2 \times 10^{-5},
$$

and so

$$
\left.\frac{\mathrm{d} N_{\gamma}}{\mathrm{d} Y_{M}}\right|_{Y_{M}=0} \simeq 190 .
$$

For the odderon-exchange cross sections, we therefore simply require the corresponding prediction for the $\gamma p$ cross sections. Unfortunately, as mentioned above, the predicted cross section is very sensitive to the treatment of the infrared QCD regime. While we will discuss some model-dependent estimates for this below, the most conservative approach is therefore simply to take the
HERA 95\% confidence upper limits on the $\pi^{0}$ and $f_{2}$ cross sections in $\gamma p$ collisions as guidance.

For the case of pseudoscalar $\eta$ production, we can use the flavor decomposition of [49]

$$
|\eta\rangle=f_{8} \cos \theta_{8}\left|q \bar{q}_{8}\right\rangle-f_{1} \sin \theta_{1}\left|q \bar{q}_{1}\right\rangle+|g g\rangle,
$$

with

$$
\begin{aligned}
& \left|q \bar{q}_{1}\right\rangle=\frac{1}{\sqrt{3}}|u \bar{u}+d \bar{d}+s \bar{s}\rangle, \\
& \left|q \bar{q}_{8}\right\rangle=\frac{1}{\sqrt{6}}|u \bar{u}+d \bar{d}-2 s \bar{s}\rangle,
\end{aligned}
$$

and

$$
\begin{array}{ll}
f_{8}=1.26 f_{\pi}, & \theta_{8}=-21.2^{\circ}, \\
f_{1}=1.17 f_{\pi}, & \theta_{1}=-9.2^{\circ} .
\end{array}
$$

While the gluonic component of course does not contribute to photoproduction, the amplitudes for the $q \bar{q}$ contributions are weighted by the quark charges, with

$$
\begin{aligned}
& \mathcal{M}_{\gamma p}^{1} \propto \frac{1}{\sqrt{3}}\left(\frac{2}{3}-\frac{1}{3}-\frac{1}{3}\right)=0 \\
& \mathcal{M}_{\gamma p}^{8} \propto \frac{1}{\sqrt{6}}\left(\frac{2}{3}-\frac{1}{3}+\frac{2}{3}\right)=\frac{1}{\sqrt{6}} .
\end{aligned}
$$

For the $\pi^{0}$ we will pick up a factor of $1 / \sqrt{2}$ from the wave function, and hence we have

$$
\frac{\sigma^{\eta}}{\sigma^{\pi^{0}}} \simeq \frac{1}{3} \frac{f_{8}^{2}}{f_{\pi}^{2}} \cos ^{2} \theta_{8}=0.46
$$

We can then scale the $\pi^{0}$ HERA limit by this factor to give the corresponding limit for the $\eta$. These results are summarized in Table I.

TABLE I. Expected upper limits for the differential production cross section at $Y_{M}=0$, of light mesons in $p \mathrm{~Pb}$ collisions, via odderon-exchange. The values are calculated using the corresponding HERA upper limits [42] on the $\pi^{0}$ and $f_{2}$ cross sections in $\gamma p$ collisions, scaled by the ion flux. The $\eta$ limit is calculated from the $\pi^{0}$ value, and scaled by the expected cross section ratio, as described in the text.

\begin{tabular}{lc}
\hline \hline $\mathrm{d} \sigma /\left.\mathrm{d} Y_{M}\right|_{Y_{M}=0}$ & Expected upper limits $[\mu \mathrm{b}]$ \\
\hline$\pi^{0}$ & 7.4 \\
$\eta$ & 3.4 \\
$f_{2}(1270)$ & 3.0 \\
\hline \hline
\end{tabular}




\section{MESON PRODUCTION VIA ODDERON EXCHANGE: CROSS SECTION CALCULATION}

In this section we consider the calculation of $\pi^{0}$ photoproduction for concreteness, but will comment on the cases of $f_{2}$ and $\eta$ meson production at the end. To calculate the cross section for $\pi^{0}$ photoproduction via odderon exchange it is most convenient to relate it to the cross section due to $\gamma \gamma$ fusion; we will discuss this background further in the following section. We have

$$
\frac{d \sigma_{\mathrm{Odd}}^{\gamma p}}{d t}=\left(\frac{9}{5}\right)^{2} \cdot 3 \cdot \frac{\left|T_{\mathrm{Odd}}(t)\right|^{2}}{\left|T_{\mathrm{QED}}(t)\right|^{2}} \cdot \frac{d \sigma_{\mathrm{QED}}^{\gamma p}}{d t} .
$$

The origin of the various terms is as follows. First, $T_{\text {Odd }}$ $\left(T_{\mathrm{QED}}\right)$ are the basic quark-quark scattering amplitudes via odderon (photon) exchange, with the quark electric charge factored out in the latter case. Then, the factor of $(9 / 5)^{2}$ accounts for the electric charge weighting of the quarks within the pion, absent in the odderon case. Next, on the proton side, at relatively large $|t|$, where the dominant contribution comes from proton $(p \rightarrow X)$ dissociation, we can roughly speaking consider our scattering process as being due to 3 independent interactions with the individual valence quarks in the proton. This gives $\sum e_{i}^{2}=1$ for the QED cross section but the number of valence quarks, i.e., a factor of 3 for the odderon cross section. ${ }^{1}$ Finally, we must consider the diagrams where the three gluons comprising the odderon couple not only to the same quark but to two quarks or each gluon to its own valence quark. This contribution contains the interference between different diagrams and strongly depends on the details of proton wave function. Neglecting interference (which is justified at large $|t|$ ) we expect in Eq. (13) an additional factor of 8 . However, the $|t|$ values we will consider are not so large, and so to be conservative we only account here for the diagram where all three gluons couple to the same quark line.

To evaluate the expected cross section due to odderon exchange we then require the $\pi^{0}$ photoproduction cross section, including proton dissociation. This can be calculated using the known $\pi^{0} \rightarrow \gamma \gamma$ width, to give

$$
\frac{d \sigma_{\mathrm{QED}}^{\gamma p}}{d t}=\frac{4 \pi \Gamma_{\gamma \gamma}}{m^{3}} \frac{\alpha}{|t|} F_{\pi}^{2}(t) F_{p}^{2}(t)=\frac{0.11 \mathrm{nb}}{|t|} F_{\pi}^{2}(t),
$$

where in the last equality we have neglected the proton form factor $F_{p}(t)$, and allow the proton to dissociate. For

\footnotetext{
${ }^{1}$ More precisely, in the case of photon exchange the inelastic photon flux from the proton is well-known [50]. However for the purposes of estimating the odderon cross section, this approximation is sufficient, while for the photon initiated background, as we will see, the suppression after imposing suitable cuts is large enough that a more precise calculation is not necessary.
}

the $t$-dependence of the pion form factor we apply the simplified $\rho$ meson pole formula

$$
F_{\pi}(t)=\frac{1}{1-t / 0.6 \mathrm{GeV}^{2}} .
$$

For the odderon exchange amplitude we have to account for the permutations of gluons in the pion-odderon vertex, that is include the diagrams where two gluons couple to the quark and the third gluon couples to the antiquark, and vice versa. Thus $T_{\text {Odd }}(t)$ takes the form

$$
\begin{aligned}
T_{\text {Odd }}(t)= & \frac{10 \alpha_{s}^{3}\left(q^{2}\right)}{81 \pi} \int\left[F_{\pi}\left(q^{2}\right)-F_{\pi}\left(4\left(q / 2-q_{1}\right)^{2}\right)\right] \\
& \times \frac{d^{2} q_{1} d^{2} q_{2} d^{2} q_{3} \delta^{(2)}\left(q-q_{1}-q_{2}-q_{3}\right)}{\left(q_{1}^{2}+\mu^{2}\right)\left(q_{2}^{2}+\mu^{2}\right)\left(q_{3}^{2}+\mu^{3}\right)}
\end{aligned}
$$

where $q_{i}$ is the transverse momentum of the $t$-channel gluon $i$ and $q^{2}=t$. The first factor accounts for the color coefficients, the identity of the 3 gluons and factors (such as $2 \pi$ ) corresponding to the Feynman loop integration. To regulate the infrared divergence associated with the loop integral we have introduced an artificial cutoff $\mu$ in the gluon propagators. The first term in the square brackets corresponds to the diagram where all three gluons couple to the same quark/antiquark, while the second term is responsible for the permutations, see e.g., Ref. [51]; due to the symmetry between the 3 gluons it is sufficient to consider only the gluon $q_{1}$ here. We can see that in the second $F_{\pi}$ term there is no form factor suppression when $q_{1}=q / 2$. In this case, both nonrelativistic quarks have half of the momentum transfer $q$, and thus go in the same direction.

Finally, to convert this to a corresponding cross section using Eq. (13) we have to multiply the amplitude $T_{\mathrm{QED}}$, which describes the QED interaction between two quarks (with the electric charges $e_{q}=1$ ), by the form factor $F_{\pi}$

$$
T_{\mathrm{QED}}=\frac{8 \pi \alpha}{t} F_{\pi}(t)
$$

that is we have to use in Eq. (13) the amplitudes of Eqs. (16) and (17).

The expected cross sections are shown in Fig. 2. We can see that while at low $t$ the QED cross section is dominant, as $t$ increases the relative contribution from odderon exchange increases. In particular, for $|t| \gtrsim 0.04 \mathrm{GeV}^{2}$, i.e., $p_{\perp}>0.2 \mathrm{GeV}$, the odderon cross section is completely dominant. Note that the average transverse momentum of the photon emitted by the ion, $p_{\perp} \sim 1 / R_{A}$, where $R_{A}$ is the ion radius, is roughly an order of magnitude smaller than this. Thus we can effectively select this larger $|t|>$ $0.04 \mathrm{GeV}^{2}$ region by placing a cut $p_{\perp}>0.2 \mathrm{GeV}$ on the produced meson. Other features of note in the figure are the rapid fall off of the odderon induced cross section as $|t|$ becomes too large, due to the large power of the running 


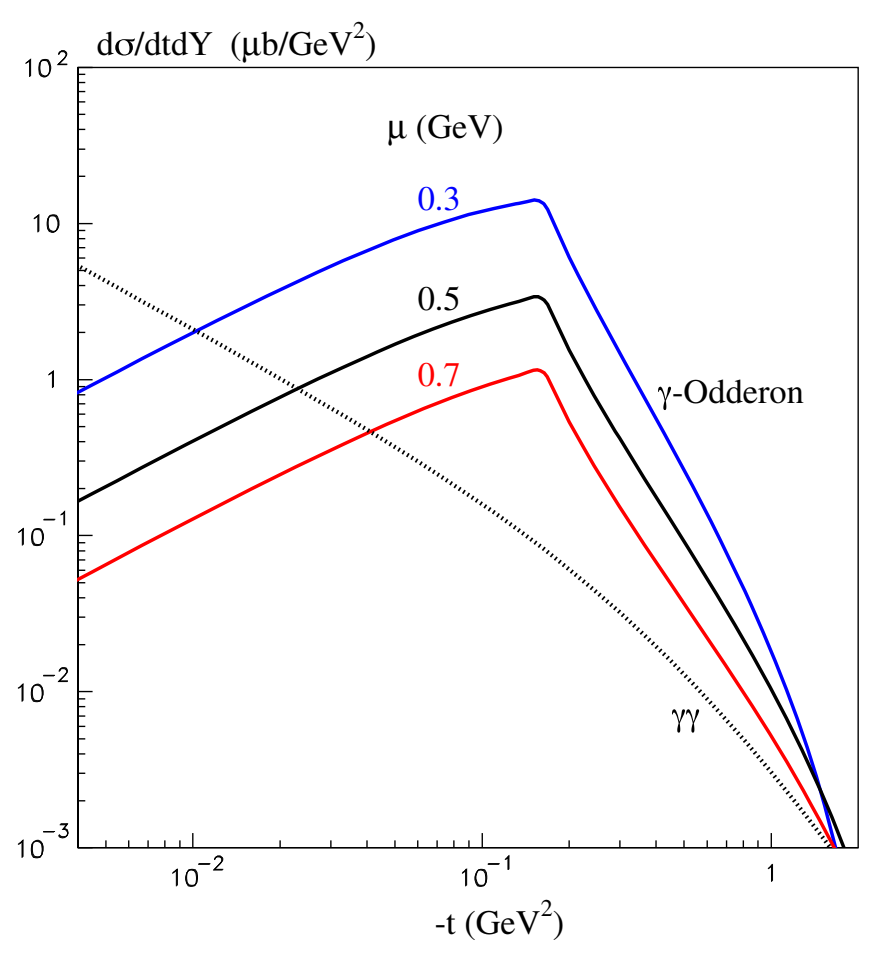

FIG. 2. Differential cross section for $\pi^{0}$ central production in proton-lead collisions due to photon-odderon (solid lines) and photon-photon (dashed line) fusion, where $\sqrt{s}_{n n}=8.16 \mathrm{TeV}$ and $Y_{M}=0$.

coupling $\sim \alpha_{s}^{6}$, and by destructive interference between the first and the second terms in square brackets of Eq. (16). In addition, the break at $|t| \simeq 0.15 \mathrm{GeV}^{2}$ is due to fact that for a smaller $|t|$ the QCD coupling was frozen at $\alpha_{s}=1$. Integrating over the range $0.04<|t|<1 \mathrm{GeV}^{2}$ (i.e., over the $0.2<p_{\perp}<1 \mathrm{GeV}$ interval) we find

$$
\sigma_{\text {Odd }}^{\gamma p}=1-12 \mathrm{nb}
$$

for $\mu=0.7-0.3 \mathrm{GeV}$.

We note that as there is no complete theoretical description of the confinement region of QCD, the above calculation only corresponds to one possibility for dealing with the infrared contribution from Eq. (16). To give an idea of the model dependence in this region, we have also examined an alternative method for dealing with the low $q_{i \perp}$ region. In particular, we explicitly limit the integral to the $q_{i}>q_{0}$ region, and in addition we replace the QCD coupling factor $\alpha_{s}(t)^{3}$ by a product of couplings evaluated at each $q_{i}^{2}$. In this case when integrating over the same $0.04<|t|<1 \mathrm{GeV}^{2}$ interval we find a smaller cross section of

$$
\sigma_{\text {Odd }}^{\gamma p}=0.07-1.6 \mathrm{nb},
$$

for $q_{0}=0.5-0.3 \mathrm{GeV}$. Interestingly in this case the relative contribution from the higher $|t|>1 \mathrm{GeV}^{2}$ region is rather large, being of roughly the same size as the above result, e.g., $\sim 1.6 \mathrm{nb}$ for $q_{0}=0.3 \mathrm{GeV}$. For the QED cross section the contribution from this large $|t|$ region is significantly lower, $\sim 0.01 \mathrm{nb}$.

We must also consider the impact of the gap survival factor, $S^{2}$. As discussed in Sec. III, the impact of additional proton-ion interactions is rather small for the ultraperipheral collision process we are considering, and thus $S_{p \mathrm{~Pb}}^{2}$ is quite close to unity. However, we must also consider the possibility of additional inelastic photon-proton and pionproton interactions. In the former case, this effect is due to the additional interaction of a $q \bar{q}$ pair created by the photon with the target proton. Unfortunately, there is no appropriate data to constrain the impact of this, and thus any estimate will be rather model dependent. Taking the $\rho$ meson dominance model of Ref. [52], we find $S_{\gamma p}^{2} \sim 0.3$, and thus we may expect the odderon signal cross section to be suppressed by a factor of $\sim 3$ relative to the results quoted above.

In summary, from the above considerations we roughly expect a signal cross section of

$$
\sigma_{\text {Odd }}^{\gamma p}=0.5-5 \mathrm{nb},
$$

for $\pi^{0}$ production, although in reality the cross section could be smaller than this, depending on the specific treatment of the infrared region.

Finally, for the $f_{2}$ meson we can apply the same procedure as above, accounting for the appropriate $\gamma \gamma$ width. For the $\eta$ we must apply the corrections as discussed in Sec. III. In both cases, we find that the cross section is expected to be roughly half that of the $\pi^{0}$.

\section{BACKGROUND FROM PHOTON-PHOTON FUSION}

The size of the background due to $\gamma \gamma$ fusion can be readily calculated using the procedure described in the previous section. In particular, we have seen from Fig. 2 that for meson $p_{\perp}>0.2 \mathrm{GeV}$, this background is expected to be very small. To quantify this, we simply apply Eq. (14), and integrate over $0.04<|t|<1 \mathrm{GeV}^{2}$. We find

$$
\sigma_{\mathrm{QED}}^{\gamma p}=0.23 \mathrm{nb} .
$$

Therefore, comparing to the estimates of the previous section, the background is expected to be under good control.

\section{BACKGROUND FROM POMERON-POMERON FUSION}

In contrast to the odderon searches at HERA via photoproduction, in heavy ion-proton collisions we must also consider the background due to pomeron-pomeron fusion, see Fig. 3. While we might naively expect such a 


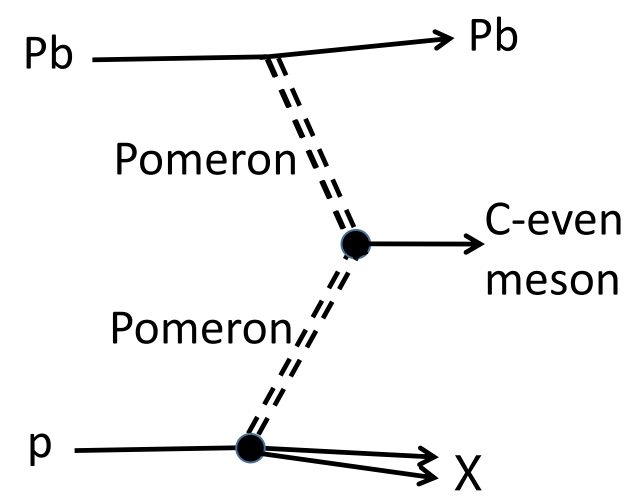

FIG. 3. A background to the odderon searches based on Fig. 1(b) arising from the production of C-even mesons by pomeron-pomeron fusion.

QCD-initiated background to be enhanced relative to odderon-induced photoproduction, in fact for the case of exclusive, or semi-exclusive production (CEP*), this is strongly suppressed by the gap survival factor $S^{2}$. In particular, in addition to the specific nucleon-nucleon collision which participates in the CEP* interaction, there is a significant probability to have additional inelastic interactions between other pairs of nucleons which will populate the rapidity gaps either side of the produced meson. It is therefore only in relatively peripheral ion- $p$ collisions that the probability of a semi-exclusive production, in which we allow for low-mass proton dissociation but no other outgoing particles except for the intact heavy ion and the C-even meson in the central region, is large enough. We first study the dependence of this contribution on the number, $A$, of nucleons in the heavy ion.

\section{A. $A$ dependence of $\mathrm{CEP} *$ cross section in $p \mathrm{~Pb}$ collisions}

For simplicity we will consider the case of genuine exclusive production, that is where the proton remains intact. To allow for the possibility of $p \rightarrow X$ low-mass dissociation we simply replace $\sigma_{\mathrm{CEP}}^{n n}$ by $\sigma_{\mathrm{CEP}}^{n n}$ in the results which follow. As we will discuss below, the transverse scale of the interaction amplitude between the proton and the nucleon in the lead ion is significantly smaller than the ion extent. This will remain a good approximation when the proton is allowed to dissociate, as here the transverse scale is very similar to the pure exclusive case. We note that the results presented below closely follow the arguments discussed in Ref. [53], and further details can be found there.

We denote the value of CEP cross section in nucleonnucleon collisions by $\sigma^{\mathrm{CEP}}$ and the total cross section as $\sigma_{\text {tot }}$. For the nucleon density in the heavy ion we use the Woods-Saxon distribution [54]

$$
\rho_{N}(r)=\frac{\rho_{0}}{1+\exp (r-R) / d},
$$

where $d$ characterizes the skin thickness and $R$ the radius of the nucleon density in the heavy ion. For ${ }^{208} \mathrm{~Pb}$ we take Refs. [55,56]

$$
\begin{aligned}
& R_{p}=6.680 \mathrm{fm}, \quad d_{p}=0.447 \mathrm{fm}, \\
& R_{n}=(6.67 \pm 0.03) \mathrm{fm}, \quad d_{n}=(0.55 \pm 0.01) \mathrm{fm} .
\end{aligned}
$$

The nucleon densities, $\rho$, are normalized to

$$
\int \rho_{p}(r) d^{3} r=Z, \quad \int \rho_{n}(r) d^{3} r=N,
$$

for which the corresponding proton (neutron) densities are $\rho_{0}=0.063(0.093) \mathrm{fm}^{-3}$.

To study those $p \mathrm{~Pb}$ processes which lead to large rapidity gaps in the final state, there are two options. Either we have incoherent semi-exclusive production with rapidity gaps around the meson, where the ion dissociates, or coherent semi-exclusive production, where it remains intact. The former can be excluded experimentally by using the zero degree calorimeter detectors, which can be used to veto on the neutral particles produced by ion dissociation. We consider both contributions below.

In the absence of survival effects, that is in the unrealistic limit of ignoring the impact of nucleon-nucleon interactions in addition to the CEP process, the incoherent cross section is simply given by

$$
\sigma_{\text {incoh }}=\int \mathrm{d}^{2} b_{\perp} T_{A}\left(b_{\perp}\right) \sigma_{\mathrm{CEP}}^{n n}=A \cdot \sigma_{\mathrm{CEP}}^{n n},
$$

where we work in the approximation that the CEP interaction can be treated as pointlike in comparison to the transverse extent of the ion. That is, the cross section for incoherent interactions between the proton and nucleons in the ion, simply scales like $\sim A$, as we would naïvely expect. Here we have introduced the transverse nuclear density

$$
T_{A}\left(b_{\perp}\right)=\int \mathrm{d} z \rho_{A}(r)=\int \mathrm{d} z\left(\rho_{n}(r)+\rho_{p}(r)\right) .
$$

To calculate the cross section due to coherent interactions we must work at amplitude level. We fix the normalization of the CEP amplitude via the condition

$$
\sigma_{\mathrm{CEP}}^{n n}=\int \mathrm{d}^{2} q_{\perp}\left|A_{\mathrm{CEP}}^{n n}\left(q_{\perp}\right)\right|^{2},
$$

where $q_{\perp}$ is the transverse momentum of the outgoing proton. Then the coherent production amplitude in protonion collisions is given by

$$
A_{\mathrm{CEP}}^{p A}\left(q_{\perp}\right)=A_{\mathrm{CEP}}^{n n}\left(q_{\perp}\right) F_{A}\left(Q^{2}\right),
$$


where the ion form factor is related to the nuclear density by

$$
F_{A}\left(Q^{2}\right)=\int \mathrm{d}^{3} r e^{i \vec{q} \cdot \vec{r}} \rho_{A}(r)
$$

in the rest frame of the ion; in this case we have $\vec{q}^{2}=-Q^{2}$. For our purposes we can simply take $Q^{2} \sim q_{\perp}^{2}$. In impact parameter space (28) corresponds to

$$
\begin{aligned}
\tilde{A}_{\mathrm{CEP}}^{p A}\left(b_{\perp}\right) & =\int \mathrm{d}^{2} b_{\perp}^{\prime} \tilde{A}_{\mathrm{CEP}}^{n n}\left(b_{\perp}^{\prime}\right) T_{A}\left(b_{\perp}-b_{\perp}^{\prime}\right), \\
& \approx T_{A}\left(b_{\perp}\right) \int \mathrm{d}^{2} b_{\perp}^{\prime} \tilde{A}_{\mathrm{CEP}}^{n n}\left(b_{\perp}^{\prime}\right) \\
& =T_{A}\left(b_{\perp}\right) A_{\mathrm{CEP}}^{n n}\left(q_{\perp}=0\right),
\end{aligned}
$$

where in the second line we have used the approximation that the size of the nucleon-nucleon amplitude is much less than the extent of the ion, i.e., $b_{\perp}^{\prime} \ll b_{\perp}$. The coherent cross section then becomes

$$
\begin{aligned}
\sigma_{\mathrm{coh}} & =\int \mathrm{d}^{2} q_{\perp}\left|A_{\mathrm{CEP}}^{p A}\left(q_{\perp}\right)\right|^{2}, \\
& =4 \pi^{2} \int \mathrm{d}^{2} b_{\perp}\left|\tilde{A}_{\mathrm{CEP}}^{p A}\left(b_{\perp}\right)\right|^{2}, \\
& \simeq 4 \pi^{2}\left|A_{\mathrm{CEP}}^{n n}\left(q_{\perp}=0\right)\right|^{2} \int \mathrm{d}^{2} b_{\perp} T_{A}\left(b_{\perp}\right)^{2}, \\
& =4 \pi \frac{\sigma_{\mathrm{CEP}}^{n n}}{\left\langle q_{\perp}^{2}\right\rangle} \int \mathrm{d}^{2} b_{\perp} T_{A}\left(b_{\perp}\right)^{2},
\end{aligned}
$$

where we have defined

$$
\left\langle q_{\perp}^{2}\right\rangle \equiv \sigma_{\mathrm{CEP}} /\left(\pi\left|A_{\mathrm{CEP}}^{n n}\left(q_{\perp}=0\right)\right|^{2}\right) .
$$

When we use that $T_{A}\left(b_{\perp}\right) \sim R$, we find that the coherent cross section scales as $T^{2} R^{2} \propto A^{4 / 3}$. This is to be expected: the short-range CEP interaction can only be coherent over the Lorentz-contracted $z$ direction, thus we only have a coherent enhancement $\sim\left(A^{1 / 3}\right)^{2}$ in that direction, rather than the $\sim A^{2}$ scaling characteristic of a long-range interaction.

We note that the above result holds for a CEP amplitude which is nonzero at $q_{\perp}=0$. However, for the production of a $0^{-}$meson the amplitude will contain an antisymmetric tensor $\epsilon^{\alpha \beta \mu \nu}$ whose indices must be saturated by the transverse momentum $q_{\perp}$, and this will therefore vanish at $q_{\perp}=0$. In other words, we would have

$$
\sigma_{\mathrm{CEP}, 0^{-}}^{n n}=\int \mathrm{d}^{2} q_{\perp} q_{\perp}^{2}\left|B_{\mathrm{CEP}}^{n n, 0^{-}}\left(q_{\perp}\right)\right|^{2},
$$

where $B_{\mathrm{CEP}}^{n n, 0^{-}}\left(q_{\perp}\right)$ is nonvanishing as $q_{\perp} \rightarrow 0$. The amplitude $\vec{A}_{\mathrm{CEP}}^{n n, 0^{-}}\left(q_{\perp}\right)=\vec{q}_{\perp} B_{\mathrm{CEP}}^{n n, 0^{-}}\left(q_{\perp}\right)$ can then be included as above via

$$
\begin{aligned}
\tilde{\vec{A}}_{\mathrm{CEP}}^{p A}\left(b_{\perp}\right) & =\int \mathrm{d}^{2} b_{\perp}^{\prime} \frac{\mathrm{d} \tilde{B}_{\mathrm{CEP}}^{n n, 0^{-}}\left(b_{\perp}^{\prime}\right)}{\mathrm{d} \vec{b}_{\perp}^{\prime}} T_{A}\left(b_{\perp}-b_{\perp}^{\prime}\right), \\
& =-\int \mathrm{d}^{2} b_{\perp}^{\prime} \tilde{B}_{\mathrm{CEP}}^{n n, 0^{-}}\left(b_{\perp}^{\prime}\right) \frac{\mathrm{d} T_{A}\left(b_{\perp}-b_{\perp}^{\prime}\right)}{\mathrm{d} \vec{b}_{\perp}^{\prime}}, \\
& \approx \frac{\mathrm{d} T_{A}\left(b_{\perp}\right)}{\mathrm{d} \vec{b}_{\perp}} B_{\mathrm{CEP}}^{n n, 0^{-}}\left(q_{\perp}=0\right),
\end{aligned}
$$

for which we have

$$
\sigma_{\mathrm{coh}}=4 \pi \frac{\sigma_{\mathrm{CEP}}^{n n}}{\left\langle q_{\perp}^{2}\right\rangle^{2}} \int \mathrm{d}^{2} b_{\perp}\left|\frac{\mathrm{d} T_{A}\left(b_{\perp}\right)}{\mathrm{d} \vec{b}_{\perp}}\right|^{2},
$$

where we define $\left\langle q_{\perp}^{2}\right\rangle^{2} \equiv \sigma_{\mathrm{CEP}} /\left(\pi\left|B_{\mathrm{CEP}}^{n n, 0^{-}}\left(q_{\perp}=0\right)\right|^{2}\right)$.

\section{B. Including the gap survival factor}

In the above expressions we have omitted the impact of accompanying nucleon-nucleon interactions to the one involved in the CEP process. These can lead to secondary particle production that will fill the rapidity gaps. The impact of this is significant, and as we will see has an important effect on the expected scaling of the CEP cross sections. To account for the survival factor $S^{2}$, that is the probability that the gaps are not filled by secondary particle production, we must multiply the integrands (25) and (35) by

$S^{2}\left(b_{\perp}\right)=\exp \left(-\sigma_{\mathrm{tot}}^{n n} \int d^{3} r^{\prime} \rho_{A}\left(r^{\prime}\right) \delta^{(2)}\left(\vec{b}_{\perp}-\vec{b}_{\perp}^{\prime}\right)\right)$,

where $\sigma_{\text {tot }}^{n n}$ is the total nucleon-nucleon cross section and $b_{\perp}^{\prime}$ is the transverse component of $r^{\prime}$; we take $\sigma_{\text {tot }}^{n n}$ rather than $\sigma_{\text {inel }}$ as even in elastic scattering the relatively large momentum transfers involved will tend to lead to ion break up.

That is, we multiply by the Poissonian probability for no additional inelastic nucleon-nucleon interactions, considering all possible nucleon positions, but with the restriction that the overall proton-ion impact parameter is fixed. In other words, we only integrate over the longitudinal component of $r$, allowing this to be simply written in terms of the ion transverse density as

$$
S^{2}\left(b_{\perp}\right)=\exp \left(-\sigma_{\mathrm{tot}}^{n n} T_{A}\left(b_{\perp}\right)\right) .
$$

Now, numerically the exponent of Eq. (43) is very large, i.e., 


$$
\sigma_{\mathrm{tot}}^{n n} T_{A}\left(b_{\perp}\right) \gg 1 \quad \text { for } r \lesssim R
$$

Thus, in this region the probability for no additional particle production is strongly (exponentially) suppressed, and the only possibility to have a non-negligible exclusive (or semiexclusive) cross section is to be close to the ion periphery, where the nucleon density and hence inelastic interaction probability is lower. We therefore expect that the scaling of e.g., Eq. (25), where all $A$ nucleons in the ion can participate equally in the CEP interaction, will significantly overestimate the predicted cross section. To be concrete, in the $r \gtrsim R$ region the nuclear density may be written as

$$
\rho(r) \approx \rho_{0} \exp (-(r-R) / d) .
$$

Then, as shown in detail in Ref. [53], the CEP cross section simply comes from a ring of radius $\sim R$ and width $\sim d$. In particular, for the incoherent cross section we have

$$
\begin{aligned}
\sigma_{\text {incoh }} & =\sigma_{\mathrm{CEP}}^{n n} \int \mathrm{d}^{2} b_{\perp} T_{A}\left(b_{\perp}\right) e^{-\sigma_{\text {tot }}^{n n} T_{A}\left(b_{\perp}\right)}, \\
& \approx \sigma_{\mathrm{CEP}}^{n n} \cdot 2 \pi R \int \mathrm{d} \delta x T_{A}\left(b_{\perp}\right) e^{-\sigma_{\text {tot }}^{n n} T_{A}\left(b_{\perp}\right)}, \\
& \sim \sigma_{\mathrm{CEP}}^{n n} \cdot 2 \pi R d, \\
& \sim \sigma_{\mathrm{CEP}}^{n n} \cdot A^{1 / 3},
\end{aligned}
$$

where in the second step we integrate over the transverse displacement $\delta x$ with respect to the ion radius $R$ in the direction of the proton-ion impact parameter. Thus we expect the incoherent cross section to scale as $A^{1 / 3}$, rather than the naïve $A$ scaling (25) of above.

For the coherent case, the expected scaling is the same, however the factor of $\left\langle q_{\perp}^{2}\right\rangle$ in (35) in principle leads to some additional numerical suppression. In particular we find that [53]

$$
\frac{4 \pi}{\sigma_{\mathrm{tot}}^{n n}\left\langle q_{\perp}^{2}\right\rangle} \sim \frac{4 \pi}{90 \mathrm{mb} \cdot 0.1 \mathrm{GeV}^{2}} \sim 0.5,
$$

where as discussed in Ref. [53] we take a rather small value of $\left\langle q_{\perp}^{2}\right\rangle \sim 0.1 \mathrm{GeV}^{2}$ to account for the impact of the nucleon-nucleon survival factor on the average $q_{\perp}$ in the CEP cross section. For the production of a $0^{-}$state, the logic is similar, but we instead apply Eq. (42). The derivative picks up a factor of $1 / d^{2}$, giving an overall factor of $1 /\left(\left\langle q_{\perp}^{2}\right\rangle d^{2}\right) \sim 1$ in comparison to the $0^{+}$case, and so we expect no significant additional suppression in this case. We note however that this only corresponds to an approximate estimate, and in particular the value of $\left\langle q_{\perp}^{2}\right\rangle$ is not universal between the even and odd parity cases. More precisely, in the odd parity case the amplitude $A^{0^{-}}$vanishes at $q_{\perp}=0$ and so the corresponding mean value of $\left\langle q_{\perp}^{2}\right\rangle$ is expected to be larger. Indeed, as we will see below, in the precise numerical calculation we do find some additional suppression in the odd parity case.

Recalling that the photoproduction cross section scales as $Z^{2}$, we can see that in the QCD-initiated case the expected scaling $\sim A^{1 / 3}$ is much milder. Thus this background, and in particular the coherent contribution, which is further numerically suppressed, may be under control. We will quantify these statements below.

\section{Double counting and the core effect}

However, before doing so there is one further complication to consider. In particular, the survival factor given by Eqs. (43) and (44) includes the possibility of additional interactions with any of the nucleons in the ion, but in the case of the active nucleon which undergoes CEP, this is already included in the value of $\sigma_{\text {CEP. }}^{n n}$. We must therefore exclude the contribution from this active nucleon in the optical density $T_{A}\left(b_{\perp}\right)$ in Eq. (44). As discussed in more detail in Ref. [53], this can be achieved by a suitable correction, removing the nucleon from the contributing outer shell of the ion and accounting for the repulsion between the nucleons by removing an interval $\pm r_{\text {core }}$ around the active nucleon from the calculation of $T_{A}\left(b_{\perp}\right)$. Here $r_{\text {core }}=0.6-0.8 \mathrm{fm}$ corresponds to the "core" region where there is a strong repulsive potential between the active nucleon and surrounding nucleons, the size of which can be estimated from the well understood deuteron case [57].

Excluding the interval $2 r_{\text {core }} \sim 1.4 \mathrm{fm}$ we find roughly a $30 \%$ correction in the power of the exponent, $\nu=\sigma_{\mathrm{tot}}^{n n} T_{A}\left(b_{\perp}\right)$. As the dominant contribution to the cross section comes from the ion periphery, where $\nu \simeq 1$, the value of the survival factor will increase by about $30-50 \%$. We will consider $r_{\text {core }}=0.6-0.8 \mathrm{fm}$ in the results below as a reasonable range of values, in the absence of a more precise description of the nucleon-nucleon correlations in the heavy-ion periphery.

\section{BACKGROUND FROM RADIATIVE DECAYS}

We in general also have to consider the background from the photoproduction of vector mesons $(J / \psi, \phi, \rho \ldots)$, followed by their radiative decay to the signal $\mathrm{C}$-meson, see Fig. 4. While not an irreducible background to the odderon signal, experimentally this may generate a contribution, as the decay photon will not always be detected. We consider this possibility explicitly in the following sections.

\section{NUMERICAL PREDICTIONS}

Table II shows the ratio of the $p \mathrm{~Pb}$ to $p p$ CEP cross sections for odd and even parity mesons produced through pomeron-pomeron fusion, calculated using the complete treatment described in detail in Ref. [53]. To evaluate the backgrounds to the odderon contribution caused by pomeron exchange in $p \mathrm{~Pb}$ collisions we then need to input the 


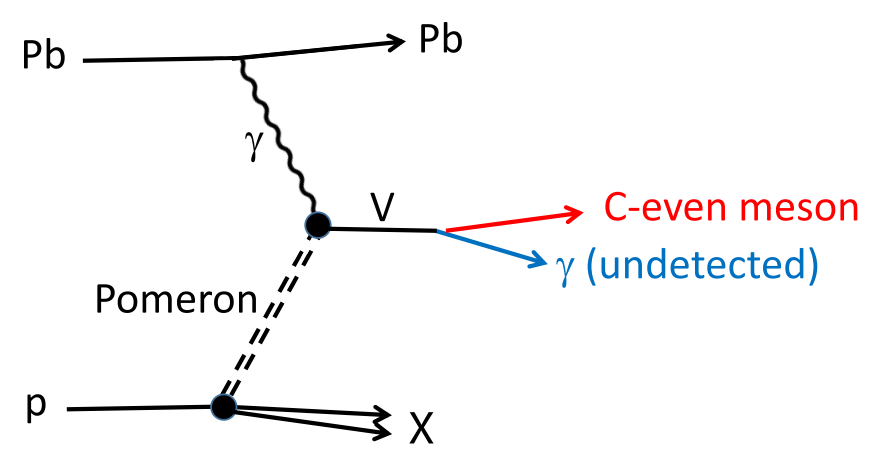

FIG. 4. A background due to the exclusive production of Ceven mesons via vector meson production followed by their radiative decay where the emitted photon is not detected.

corresponding cross sections in $p p$ collisions. Theoretically, these are not straightforward to predict precisely, and so it is more reliable to take the existing experimental measurements of these $p p$ processes as a guide. We will for concreteness quote all results for central production in the $\left|Y_{M}\right|<1$ rapidity region, that is integrated over 2 units of the meson rapidity in the central region. Although we do not consider it explicitly here, this should roughly correspond to the expectations for the LHCb detector, i.e., with $2<Y_{M}<4.5$.

\section{A. $f_{2}$ production}

For the case of $f_{2}$ production, the semi-exclusive process (i.e., including an admixture due to proton dissociation) has been measured by CMS [58] at $7 \mathrm{TeV}$. Using this information we can, to a good approximation, estimate the rate at $8.16 \mathrm{TeV}$ collision energy relevant for $p \mathrm{~Pb}$ collisions. We find for $\left|Y_{M}\right|<1$ that

$$
\sigma_{p p}^{f_{2}}=2-3 \mu \mathrm{b} .
$$

From Table II we can see that his corresponds to roughly

$$
\sigma_{p \mathrm{~Pb}}^{f_{2}, \mathrm{IP}}=5-9 \mu \mathrm{b}, \quad \sigma_{p \mathrm{~Pb}^{*}}^{f_{2}, \mathrm{IP}}=9-16 \mu \mathrm{b},
$$

where $\mathrm{Pb}^{*}$ indicates the semi-exclusive (incoherent) cross section, i.e., where the lead ion does not remain intact. We can see from Table I that the expected upper limit to the odderon-initiated cross section is

TABLE II. The ratio $\sigma_{p \mathrm{~Pb}} / \sigma_{p p}$ for exclusive and semi-exclusive CEP of odd $\left(0^{-}\right)$and even $\left(0^{+}, 2^{+}\right)$parity mesons, produced through pomeron-pomeron fusion at $\sqrt{s_{n n}}=5.02 \mathrm{TeV}$, shown for two values of $r_{\text {core }}$.

\begin{tabular}{lllll}
\hline \hline & \multicolumn{2}{c}{ Exclusive } & \multicolumn{2}{c}{ Semi-exclusive } \\
\hline$r_{\text {core }}(\mathrm{fm})$ & 0.8 & 0.6 & 0.8 & 0.6 \\
$\mathrm{P}$ even & 2.5 & 3.0 & 4.7 & 5.3 \\
$\mathrm{P}$ odd & 0.45 & 0.5 & 4.7 & 5.3 \\
\hline \hline
\end{tabular}

$$
\sigma_{p \mathrm{~Pb}}^{f_{2}, \mathrm{O}}<6.0 \mu \mathrm{b}
$$

at the $95 \%$ confidence level, where we have integrated over the interval $\left|Y_{M}\right|<1$. Thus, unfortunately even considering only the purely exclusive case (i.e., with vetoes applied on the lead ion dissociation) the predicted background is somewhat larger than the experimental upper limit, and so this will be a very challenging channel in which to observe odderon exchange.

The background due to vector meson radiative decay is rather small. The main contribution comes from the $J / \psi \rightarrow$ $\gamma f_{2}$ decay, with branching ratio $1.4 \times 10^{-3}$ [2]. Accounting for the $J / \psi$ photoproduction cross section [59] this gives a background in $p-\mathrm{Pb}$ collisions of only $\sim 0.02 \mu \mathrm{b}$.

Note that we may improve the Odderon to PomeronPomeron background ratio by selecting events with proton break-up since we expect a larger probability of break-up in the case of Odderon exchange [32].

\section{B. $\pi^{0}$ production}

The $\pi^{0}$ meson cannot be produced by pomeron-pomeron fusion due to isospin conservation. This background will therefore be absent, and from Table I we see that the expected upper limit on the odderon-initiated cross section is

$$
\sigma_{p \mathrm{~Pb}}^{\pi^{0}, \mathrm{O}}<14.8 \mu \mathrm{b}
$$

for $\left|Y_{M}\right|<1$. In principle the situation looks much more favorable in this case, although, in practice, the observation of an exclusive $\pi^{0}$ signal could be difficult for various experimental reasons. For example, the trigger conditions for the detection of soft photons are challenging and the presence of additional $\pi^{0}$ mesons in the final state in the CEP* process could complicate the signal. We note that as the pion cannot be produced via pomeron-pomeron fusion, the contribution from events with ion dissociation should already be small, and so there is no further benefit in applying a cut using the Zero Degree Calorimeter.

In addition to this there is a large background from $\omega$ meson production, followed by the radiative $\omega \rightarrow \gamma \pi^{0}$ decay. Taking a $\omega$ photoproduction cross section of about $2 \mu \mathrm{b}$ [59] and a branching ratio of $8 \%$ [2], we expect a total background cross section of $60 \mu \mathrm{b}$, that is roughly four times as large as the expected upper limit (55) of the signal. On the other hand, the background from ultraperipheral $\omega$ production where the additional photon from the radiative decay is detected can be measured and this background subtracted in a reasonably data-driven way. However, the overall size of the background suggests this will need a relatively careful treatment.

\section{C. $\eta$ production}

For the $\eta(548)$ meson, the dominant $S U(3)$-flavor octet component will, as in the $\pi^{0}$ case, not couple to the double 
pomeron exchange production channel. This background will not be entirely absent, due to the nonzero $S U(3)$-flavor singlet component of the $\eta$, however this contribution will be suppressed by the relatively small mixing angle [2]

$$
\sigma_{p \mathrm{~Pb}}^{\eta, \mathrm{IP}} \propto \sin ^{2}\left(\theta_{1}\right)=0.03-0.1
$$

that is, by at least an order of magnitude in comparison to $f_{2}$ production. However, in addition to this, we expect a further suppression due to the $J^{P}=0^{-}$nature of the $\eta$ meson, the production of which is typically suppressed in double pomeron exchange [60]. In particular, the $\eta$ production vertex must be symmetric with respect to the two pomeron exchanges and simultaneously contain an antisymmetric tensor, due to the odd parity of the meson. The simplest expression satisfying these requirements is

$$
V \propto \epsilon_{\alpha \beta \mu \nu} k_{1 L}^{\alpha} k_{2 L}^{\beta} k_{1 \perp}^{\mu} k_{2 \perp}^{\nu},
$$

where $k_{1 t}, k_{2 t}$ and $k_{1 L}, k_{2 L}$ are the transverse and longitudinal momenta exchanged through the pomerons. We therefore have

$$
\frac{\sigma_{p \mathrm{~Pb}}^{\eta, \mathrm{IP}}}{\mathrm{d}^{2} k_{1 \perp} \mathrm{d}^{2} k_{2 t}} \propto k_{1 \perp}^{2} k_{2 \perp}^{2}
$$

where these factors of $k_{\perp}^{2}$ are absent in the even parity case. The dimension of these factors must be compensated by some additional scale. While we do not know the precise value of this scale for such a nonperturbative process, we can expect it driven by the pomeron size, that is by the value of $\alpha_{\mathrm{IP}}^{\prime}$, i.e., the slope of pomeron trajectory. Phenomenologically, $\alpha_{\mathrm{IP}}^{\prime} \sim 0.25 \mathrm{GeV}^{2}$ is rather small, and so we expect

$$
\sigma_{p \mathrm{~Pb}}^{\eta, \mathrm{IP}} \propto \sin ^{2}\left(\theta_{1}\right)\left(\left\langle k_{\perp}^{2}\right\rangle \alpha_{\mathrm{IP}}^{\prime}\right)^{2} \lesssim 10^{-3}
$$

where we have taken the rather conservative values of $\theta_{1} \sim$ $20^{\circ}$ and $\left\langle k_{\perp}^{2}\right\rangle=0.5 \mathrm{GeV}^{2}$; that is, the suppression may be significantly stronger. For example, if we take the value of $\theta_{1} \sim 10^{\circ}$ preferred by Ref. [49], then the suppression is about 4 times larger. Taking the observed $f_{2}$ cross section as a baseline, we therefore roughly expect the background due to double pomeron exchange to be $O(0.01) \mu \mathrm{b}$, and potentially much smaller than this. From Table I we see that this is significantly less than the predicted upper limit on the odderon-initiated cross section (for $\left|Y_{M}\right|<1$ ) of

$$
\sigma_{p \mathrm{~Pb}}^{\eta, O} \lesssim 6.8 \mu \mathrm{b}
$$

Finally, we have to consider the background due to radiative vector meson decay, in particular from $\phi \rightarrow \gamma \eta$ when the relatively soft additional photon escapes detection. With $\sigma(\gamma p \rightarrow \phi+p)=1 \mu \mathrm{b}$ [59] and $1.3 \%$ branching [2] we expect a total background cross section of about $5 \mu \mathrm{b}$, that is smaller than but comparable to the upper limit on the signal. We in addition expect a total background cross section of $\sim 1.2 \mu \mathrm{b}$ from $\rho \rightarrow \gamma \eta$ decay. In both cases by measuring the corresponding backgrounds where the additional photons are detected, we should be able to subtract these, but again some care is clearly needed. Finally there is in principle a contribution coming from $\eta^{\prime}$ production in pomeron-pomeron fusion, followed by the $\eta^{\prime} \rightarrow \eta \pi^{0} \pi^{0}$ decay. However, we would expect this to be efficiently rejected by observing at least one of four photons from $\pi^{0}$ decay in the final state.

\section{D. $\eta_{c}$ production}

Theoretically, $\eta_{c}$ photoproduction has the advantage that due to the relatively large charm quark mass, $m_{c}$, the perturbative QCD calculation of the odderon-meson coupling is more justified, although the overall amplitude is nonetheless sensitive to the infrared region due to the modelling of the odderon-proton coupling. On the other hand, due to the larger meson mass the cross section is much smaller. In Refs. [61-64] this was estimated to be $\sigma\left(\gamma p \rightarrow \eta_{c}+X\right) \sim 60 \mathrm{pb}$ in total, and about $10 \mathrm{pb}$ for $|t|>1 \mathrm{GeV}^{2}$. That is we expect

$$
\frac{d \sigma}{d y}=2-10 \mathrm{nb},
$$

in $p \mathrm{~Pb}$ collisions. The background from pomeron-pomeron fusion was calculated in Ref. [65] to be roughly

$$
\sigma_{p p}^{\eta_{c}}=0.4 \mathrm{nb},
$$

at $\sqrt{s}=7-14 \mathrm{TeV}$, corresponding to

$$
\sigma_{p \mathrm{~Pb}}^{\eta_{c}, \mathrm{IP}}=0.2 \mathrm{nb}, \quad \sigma_{p \mathrm{~Pb}^{*}}^{\eta_{c}, \mathrm{IP}}=2 \mathrm{nb},
$$

where the uncertainty in the PQCD prediction is larger $(\sim \underset{\div}{\sim})$ than the spread in Table II and we therefore only quote the approximate central values for illustration. This is therefore smaller than the expected odderon signal, in particular for the case of no ion dissociation. Clearly a measurement of $\eta_{c}$ production in the $p p$ mode would further help calibrate this background.

Unfortunately, the branching ratios of accessible $\eta_{c}$ decay channels are all rather small. The most convenient modes for experimental observation are [2]:

$$
\eta_{c} \rightarrow \rho \rho: \quad(1.8 \pm 0.5) \%,
$$

where the branching ratio is given. This corresponds to $0.6 \%$ in the $2 \rho^{0} \rightarrow 2\left(\pi^{+} \pi^{-}\right)$mode. In addition we have

$$
\eta_{c} \rightarrow K^{* 0} K^{-} \pi^{+}: \quad(2.0 \pm 0.7) \%,
$$


giving about $1.3 \%$ in the $K^{-} K^{+} \pi^{+} \pi^{-}$mode, and

$$
\eta_{c} \rightarrow K \bar{K} \pi: \quad(7.3 \pm 0.5) \%
$$

which can be observed via the $K_{s}^{0} \pi^{+} K^{-}$and $K_{s}^{0} \pi^{-} K^{+}$ channels for which we get $\mathrm{a} \simeq 1.7 \%$ branching. Finally we have

$$
\eta_{c} \rightarrow 3\left(\pi^{+} \pi^{-}\right): \quad(1.8 \pm 0.4) \% .
$$

Summing over all of the charged decay modes, we find a total branching ratio of $\sim 5 \%$, corresponding to an expected odderon signal of $\sim 0.1-0.5 \mathrm{nb}$. The cross section alone is certainly large enough to be experimentally feasible, however it is very challenging to separate such a signal from minimum bias events, where the cross section $(\sim 1 \mathrm{~b})$ is more than a factor $\sim 10^{9}$ larger. To bypass this, it would therefore be essential to select ultraperipheral events with Large Rapidity Gaps at the trigger stage.

A potentially even more serious issue arises due to the background from $J / \psi \rightarrow \gamma \eta_{c}$ decay, which occurs with branching $1.7 \%$ [2]. This corresponds to a total background cross section of $\sim 250 \mathrm{nb}$, excluding the $\eta_{c}$ branchings, that is more than 20 times larger than the expected odderon induced cross section. Clearly accounting for such a background will be highly challenging.

\section{E. Summary of numerical predictions}

We draw together in Table III the results for the odderon signal and backgrounds for the various $\mathrm{C}$-even mesons, $M$, discussed above. The first column shows the upper limit of the cross section for the odderon-exchange process $(\mathrm{Pb} p \rightarrow \mathrm{Pb} M X)$ deduced from the HERA data for $(\gamma p \rightarrow M X)$ as described in Sec. III and listed in Table I. The other columns give the cross sections expected for the odderon signal and background in the (semi) exclusive process

$$
\mathrm{Pb} p \rightarrow \mathrm{Pb}+M+X,
$$

where here the + signs denote the presence of large rapidity gaps. Note the Table shows $d \sigma / d Y_{M}$ at $Y_{M}=0$ while the text presents numbers for $\sigma$ integrated over the central interval $\left|Y_{M}\right|<1$, so the cross section is essentially twice as large.

\section{CONCLUSIONS AND OUTLOOK}

In this paper we have discussed the possibility of observing odderon exchange in ultraperipheral $p \mathrm{~Pb}$ collisions. We have shown that the signal cross sections for the semi-exclusive production of $\mathrm{C}$-even light mesons due to odderon exchange could be quite large, up to the $\mu \mathrm{b}$ level, and so represent a viable search channel. However, it is important to emphasise that an experimentally feasible signal cross section is a necessary but not sufficient condition when searching for the so-far rather elusive QCD odderon. In particular, it is essential to consider the purity of the expected signal, and to quantitatively estimate the contribution from all potential backgrounds. This has been the main goal of this paper.

We have in particular considered the two principle irreducible backgrounds, due to photon-photon and pomeron-pomeron fusion. While in $A A$ collisions the contributions from the former, which is enhanced by $\sim Z^{4}$ will be overwhelming, in $p p$ collisions the contribution from the latter will be strongly dominant. We have therefore identified $p A$ collisions, specifically $p-\mathrm{Pb}$ in the context of the LHC, as the most promising channel. In this case, we have found that the photon-photon background can be strongly suppressed by requiring that an appropriate cut $\left(p_{\perp} \gtrsim 0.2 \mathrm{GeV}\right)$ is placed on the produced meson, while leaving the signal roughly unchanged. The background from pomeron-pomeron fusion is found to scale quite mildly with mass number $\sim A^{1 / 3}$, due to the suppression induced by requiring that no additional particles be produced. This leads the background, which naively we might expect to be overwhelming, to be of the same order or even smaller than the odderon-exchange signal.

We have considered several candidate C-even mesons with which to observe odderon exchange: the $\pi^{0}, \eta(548), \eta_{c}$ and $f_{2}(1270)$. For the C-even $f_{2}$ meson there is very low background due to the vector meson decay (like $J / \psi \rightarrow \gamma f_{2}$ ) while the background due to double pomeron exchange is found to be somewhat larger than any possible

TABLE III. The expected cross sections $\left(d \sigma / d Y_{M}\right.$ at $Y_{M}=0$ in $\left.\mu \mathrm{b}\right)$ of the odderon signal and backgrounds in the CEP* ultraperipheral production of C-even mesons $(M)$ in high-energy proton-lead collisions

\begin{tabular}{|c|c|c|c|c|c|}
\hline \multirow[b]{2}{*}{ C-even meson $(M)$} & \multicolumn{2}{|c|}{ Odderon Signal } & \multicolumn{3}{|c|}{ Backgrounds } \\
\hline & Upper Limit & QCD Prediction & $\gamma \gamma$ & Pomeron-pomeron & $V \rightarrow M+\gamma$ \\
\hline$\overline{\pi^{0}}$ & 7.4 & $0.1-1$ & 0.044 & $\ldots$ & 30 \\
\hline$f_{2}(1270)$ & 3 & $0.05-0.5$ & 0.020 & $3-4.5$ & 0.02 \\
\hline$\eta(548)$ & 3.4 & $0.05-0.5$ & 0.042 & Negligible & 3 \\
\hline$\eta_{c}$ & $\ldots$ & $(0.1-0.5) \times 10^{-3}$ & 0.0025 & $\sim 10^{-5}$ & 0.012 \\
\hline
\end{tabular}
$(\mathrm{Pb}+p \rightarrow \mathrm{Pb}+M+X)$ integrated over the interval $0.2<p_{\perp}<1 \mathrm{GeV}$. In the $\eta_{c}$ case a total branching ratio of 0.05 has been applied, i.e., summing over the channels discussed in the text. 
signal, in particular taking into account existing HERA upper limits. In principle, the signal-to-pomeron background ratio could be greatly improved by selecting events with very small outgoing ion transverse momenta (however this is not experimentally feasible in the present LHC experiments) or to select events with proton break-up. On the other hand, the production of the $S U(3)$ flavor octet $\pi^{0}$ is forbidden in double pomeron exchange, and so such a background is entirely absent, although this may prove to be an experimentally challenging channel. Moreover here we have a larger reducible background due to radiative $\omega$ decay, $\omega \rightarrow \pi^{0} \gamma$. For the $\eta(548)$, the relatively small flavor singlet component combined with the suppression expected due to the odd parity of the meson leads us to expect the pomeronpomeron background to be many orders of magnitude below the odderon-initiated signal. Unfortunately in this case the reducible contributions coming from $\phi \rightarrow \gamma \eta$ and $\eta^{\prime} \rightarrow$ $\eta \pi^{0} \pi^{0}$ decays are again rather large. Finally, the production of the heavier $\eta_{c}$, while in principle representing a viable channel, has a much smaller production cross section and the signal will also be reduced by the small branching ratios of the experimentally accessible decay channels. The results are summarized in Table III.

Our study is not intended to be exhaustive, and the production of heavier mesons such as the $\chi_{c}$, or light mesons such as the $f_{0}(980), f_{1}(1285), f_{1}(1420), \eta(1405)$ and $\eta(1475)$, though not considered explicitly here, may also be worth exploring. In particular the $f_{1}(1285)$ has the advantage of a relatively small width and the fact that due to its $J^{P}=1^{+}$quantum numbers the backgrounds due to pomeron-pomeron and/or $\gamma \gamma$ fusion should be suppressed.

To conclude, our study highlights the importance of considering the background contributions in the case of observing odderon exchange via photoproduction in $p-\mathrm{Pb}$ collisions at the LHC. In particular, while we find that the expected signal is certainly sufficiently large to be observed at the LHC, these backgrounds are not always negligible and can pose a challenge. Here we have discussed various techniques for bringing the backgrounds under control, paving the way for future experimental searches at the LHC.

\section{ACKNOWLEDGMENTS}

We thank Ronan McNulty and E. L. Kryshen for the interest to this work and for useful discussions. We are grateful to Vadim Isakov for the discussion of the proton and neutron distributions in a heavy nucleus. L. H. L. thanks the Science and Technology Facilities Council (STFC) for support via Grant Awards No. ST/L000377/1 and No. ST/P004547/1. M. G. R. thanks the IPPP at the University of Durham for hospitality. V. A. K. acknowledges support from a Royal Society of Edinburgh Auber award.
[1] G. Antchev et al. (TOTEM Collaboration), First determination of the $\rho$ parameter at $\sqrt{s}=13 \mathrm{TeV}$ probing the existence of a colorless three-gluon bound state, CERN Report No. CERN-EP-2017-335, 2017, arXiv:1812.08610.

[2] C. Patrignani et al. (Particle Data Group), Review of particle physics, Chin. Phys. C 40, 100001 (2016).

[3] E. Martynov and B. Nicolescu, Did TOTEM experiment discover the odderon?, Phys. Lett. B 778, 414 (2018).

[4] V. A. Khoze, A. D. Martin, and M. G. Ryskin, Black disk, maximal odderon and unitarity, Phys. Lett. B 780, 352 (2018).

[5] V. A. Khoze, A. D. Martin, and M. G. Ryskin, Elastic proton-proton scattering at $13 \mathrm{TeV}$, Phys. Rev. D 97, 034019 (2018).

[6] V. P. Gonalves and B. D. Moreira, $\eta_{c}$ production in photoninduced interactions at the LHC, Phys. Rev. D 97, 094009 (2018).

[7] V. P. Goncalves, Searching the odderon in the diffractive $f_{2}(1270)$ photoproduction at $p A$ collisions, arXiv:1811 .07622 .

[8] E. Martynov and B. Nicolescu, Evidence for maximality of strong interactions from LHC forward data, Phys. Lett. B 786, 207 (2018).
[9] V. A. Khoze, A. D. Martin, and M. G. Ryskin, Elastic and diffractive scattering at the LHC, Phys. Lett. B 784, 192 (2018).

[10] Y. M. Shabelski and A. G. Shuvaev, Real part of $p p$ scattering amplitude in Additive Quark Model at LHC energies, Eur. Phys. J. C 78, 497 (2018).

[11] M. Broilo, E. G.S. Luna, and M.J. Menon, Leading pomeron Contributions and the TOTEM Data at $13 \mathrm{TeV}$, in 14th International Workshop on Hadron Physics (Hadron Physics 2018) Florianopolis, Santa Catarina, Brazil, 2018 (2018), arXiv:1803.06560.

[12] M. Broilo, E. G.S. Luna, and M. J. Menon, Soft pomerons and the forward LHC data, Phys. Lett. B 781, 616 (2018).

[13] S. M. Troshin and N. E. Tyurin, Implications of the $\rho(s)$ measurements by TOTEM at the LHC, Mod. Phys. Lett. A 33, 1850206 (2018).

[14] W. Broniowski, L. Jenkovszky, E. Ruiz Arriola, and I. Szanyi, Hollowness in $p p$ and $p \bar{p}$ scattering in a Regge model, Phys. Rev. D 98, 074012 (2018).

[15] T. Csorgo, R. Pasechnik, and A. Ster, odderon and proton substructure from a model-independent Lévy imaging of elastic $p p$ and $p \bar{p}$ collisions, arXiv:1807.02897. 
[16] E. Martynov and B. Nicolescu, odderon effects in the differential cross-sections at Tevatron and LHC energies, arXiv:1808.08580.

[17] N. Bence, L. Jenkovszky, and I. Szanyi, New physics from TOTEM's recent measurements of elastic and total cross sections, arXiv:1808.03588.

[18] I. M. Dremin, Several effects unexplained by QCD, Universe 4, 65 (2018).

[19] O. V. Selyugin and J. R. Cudell, odderon, HEGS model and LHC data, in diffraction and low-x 2018 (Difflowx2018) Reggio Calabria, Italy, 2018 (2018), arXiv:1810.11538.

[20] L. Lukaszuk and B. Nicolescu, A possible interpretation of $\mathrm{p}$ p rising total cross-sections, Lett. Nuovo Cimento 8, 405 (1973).

[21] D. Joynson, E. Leader, B. Nicolescu, and C. Lopez, NonRegge and HyperRegge effects in Pion-Nucleon charge exchange scattering at high-energies, Nuovo Cimento A 30, 345 (1975).

[22] M. A. Braun, Odderon and QCD, arXiv:hep-ph/9805394.

[23] C. Ewerz, The odderon in quantum chromodynamics, arXiv:hep-ph/0306137.

[24] C. Ewerz, The odderon: Theoretical status and experimental tests, in 11th International Conference on Elastic and Diffractive Scattering: Towards High Energy Frontiers: The 20th Anniversary of the Blois Workshops, 17th Rencontre de Blois (EDS 05) Chateau de Blois, Blois, France, 2005 (2005).

[25] C. Ewerz, Searching for the odderon at HERA and the LHC, in Proceedings, HERA and the LHC Workshop Series on the Implications of HERA for LHC Physics: 2006-2008 (2009), pp. 510-515.

[26] J. Kwiecinski and M. Praszalowicz, Three Gluon integral equation and Odd c singlet Regge singularities in QCD, Phys. Lett. 94B, 413 (1980).

[27] J. Bartels, High-energy behavior in a nonabelian gauge theory (II), Nucl. Phys. B175, 365 (1980).

[28] B. L. Ioffe, V. S. Fadin, and L. N. Lipatov, Quantum Chromodynamics: Perturbative and Nonperturbative Aspects, Vol. 30 (Cambridge University Press, Cambridge England, 2010).

[29] J. Bartels, L. N. Lipatov, and G. P. Vacca, A new odderon solution in perturbative QCD, Phys. Lett. B 477, 178 (2000).

[30] M. M. Block, Hadronic forward scattering: Predictions for the large hadron collider and cosmic rays, Phys. Rep. 436, 71 (2006).

[31] M. G. Ryskin, Odderon and polarization phenomena in QCD, Sov. J. Nucl. Phys. 46, 337 (1987).

[32] E. M. Levin and M. G. Ryskin, High-energy hadron collisions in QCD, Phys. Rep. 189, 268 (1990).

[33] J. Finkelstein, H. M. Fried, K. Kang, and C. I. Tan, Forward scattering at collider energies and Eikonal Unitarization of odderon, Phys. Lett. B 232, 257 (1989).

[34] V. V. Barakhovsky, I. R. Zhitnitsky, and A. N. Shelkovenko, Odderon: A sharp signal at HERA, Phys. Lett. B 267, 532 (1991).

[35] M. G. Ryskin, Exclusive f2 leptoproduction via the odderon exchange, Eur. Phys. J. C 2, 339 (1998).

[36] W. Kilian and O. Nachtmann, Single pseudoscalar meson production in diffractive e p scattering, Eur. Phys. J. C 5, 317 (1998).
[37] M. Rueter, H. G. Dosch, and O. Nachtmann, Odd CP contributions to diffractive processes, Phys. Rev. D 59, 014018 (1998).

[38] E. R. Berger, A. Donnachie, H. G. Dosch, W. Kilian, O. Nachtmann, and M. Rueter, Odderon and photon exchange in electroproduction of pseudoscalar mesons, Eur. Phys. J. C 9, 491 (1999).

[39] E. R. Berger, A. Donnachie, H. G. Dosch, and O. Nachtmann, Observing the odderon: Tensor meson photoproduction, Eur. Phys. J. C 14, 673 (2000).

[40] I. P. Ivanov, N. N. Nikolaev, and I. F. Ginzburg, Possible odderon discovery at HERA via charge asymmetry in the diffractive $\pi^{+} \pi^{-}$production, Sci. Cult. Ser.-Phys. 21, 728 (2002).

[41] A. Bzdak, L. Motyka, L. Szymanowski, and J. R. Cudell, Exclusive $J / \psi$ and $\gamma$ hadroproduction and the QCD odderon, Phys. Rev. D 75, 094023 (2007).

[42] J. Olsson (H1 Collaboration), Search Odderon induced contributions to exclusive meson photoproduction at HERA, in New Trends in High-Energy Physics: Experiment, Phenomenology, Theory, Proceedings, International Conference, Yalta, Crimea, Ukraine, 2001 (2001), pp. 79-87.

[43] C. Adloff et al. (H1 Collaboration), Search for odderon induced contributions to exclusive $\pi^{0}$ photoproduction at HERA, Phys. Lett. B 544, 35 (2002).

[44] T. Berndt (H1 Collaboration), Investigation of pomeron and odderon induced photoproduction of mesons decaying to pure multiphoton final states at HERA, Acta Phys. Pol. B 33, 3499 (2002).

[45] M. Rueter and H. G. Dosch, Nucleon structure and highenergy scattering, Phys. Lett. B 380, 177 (1996).

[46] E. Fermi, On the theory of the impact between atoms and electrically charged particles, Z. Phys. 29, 315 (1924).

[47] L. D. Landau and E. M. Lifschitz, Production of electrons and positrons by a collision of two particles, Phys. Z. Sowjetunion 6, 244 (1934).

[48] V. M. Budnev, I. F. Ginzburg, G. V. Meledin, and V. G. Serbo, The two photon particle production mechanism. Physical problems. Applications. Equivalent photon approximation, Phys. Rep. 15, 181 (1975).

[49] T. Feldmann, P. Kroll, and B. Stech, Mixing and decay constants of pseudoscalar mesons, Phys. Rev. D 58, 114006 (1998).

[50] A. Manohar, P. Nason, G. P. Salam, and G. Zanderighi, How Bright is the Proton? A Precise Determination of the Photon Parton Distribution Function, Phys. Rev. Lett. 117, 242002 (2016).

[51] E. M. Levin and M. G. Ryskin, Born approximation of QCD for description of high-energy hadronic interactions, Sov. J. Nucl. Phys. 34, 619 (1981).

[52] A. B. Kaidalov, V. A. Khoze, A. D. Martin, and M. G. Ryskin, Factorization breaking in diffractive dijet photoproduction at HERA, Eur. Phys. J. C 66, 373 (2010).

[53] L. A. Harland-Lang, V. A. Khoze, and M. G. Ryskin, Exclusive LHC physics with heavy ions: SuperChic 3, Eur. Phys. J. C 79, 39 (2019).

[54] R. D. Woods and D. S. Saxon, Diffuse surface optical model for nucleon-nuclei scattering, Phys. Rev. 95, 577 (1954).

[55] C. M. Tarbert et al., Neutron Skin of ${ }^{208} \mathrm{~Pb}$ from Coherent Pion Photoproduction, Phys. Rev. Lett. 112, 242502 (2014). 
[56] A. B. Jones and B. A. Brown, Two-parameter Fermi function fits to experimental charge and point-proton densities for Pb208, Phys. Rev. C 90, 067304 (2014).

[57] R. V. Reid, Jr., Local phenomenological nucleon-nucleon potentials, Ann. Phys. (N.Y.) 50, 411 (1968).

[58] V. Khachatryan et al. (CMS Collaboration), Exclusive and semi-exclusive $\pi^{+} \pi^{-}$production in proton-proton collisions at $\sqrt{s}=7 \mathrm{TeV}$, arXiv:1706.08310.

[59] K. C. Voss (ZEUS and H1 Collaboration), Vector meson production at HERA, in Proceedings, 38th Rencontres de Moriond on QCD and High-Energy Hadronic Interactions: Les Arcs, France, 2003 (2003).

[60] A. B. Kaidalov, V. A. Khoze, A. D. Martin, and M. G. Ryskin, Central exclusive diffractive production as a spin-parity analyser: From Hadrons to Higgs, Eur. Phys. J. C 31, 387 (2003).
[61] J. Bartels, M. A. Braun, and G. P. Vacca, The process $\gamma(*)+p \rightarrow \eta_{c}+X$ : A test for the perturbative QCD odderon, Eur. Phys. J. C 33, 511 (2004).

[62] J. Czyzewski, J. Kwiecinski, L. Motyka, and M. Sadzikowski, Exclusive $\eta_{c}$ photoproduction and electroproduction at HERA as a possible probe of the odderon singularity in QCD, Phys. Lett. B 398, 400 (1997).

[63] J. P. Ma, Diffractive photoproduction of eta(c), Nucl. Phys. A727, 333 (2003).

[64] R. Engel, D. Yu. Ivanov, R. Kirschner, and L. Szymanowski, Diffractive meson production from virtual photons with odd charge-parity exchange, Eur. Phys. J. C 4, 93 (1998).

[65] L. A. Harland-Lang, V. A. Khoze, M. G. Ryskin, and W. J. Stirling, Central exclusive production within the Durham model: A review, Int. J. Mod. Phys. A 29, 1430031 (2014). 